Pacific

Journal of

Mathematics

CANONICAL BASES AND THE CONJUGATING REPRESENTATION OF A SEMISIMPLE GROUP

Pierre Baumann 


\title{
CANONICAL BASES AND THE CONJUGATING REPRESENTATION OF A SEMISIMPLE GROUP
}

\author{
PierRe BAUmanN
}

\begin{abstract}
Let $G$ be a semisimple simply connected affine algebraic group over an algebraically closed field $k$ of characteristic zero, let $A(G)$ be the $k$-algebra of regular functions of $G$, and let $C(G)$ be the subalgebra consisting of class functions. We explain how Lusztig's work on canonical bases affords a constructive proof of the fact, due to Richardson, that $A(G)$ is a free $C(G)$-module.
\end{abstract}

\section{Introduction.}

We fix an algebraically closed field $k$ of characteristic zero. Let $G$ be a reductive affine algebraic group over $k$ and let $V$ be an affine $G$-variety over $k$. We denote by $A(G)$ and $A(V)$ the $k$-algebras of regular functions on $G$ and $V$ respectively. The action of $G$ on $V$ gives rise to a rational representation of $G$ on $A(V)$. A natural question is to investigate whether the algebra $A(V)$ is a free module over its subalgebra $A(V)^{G}$ of invariant elements. The case where $V$ is a $k$-vector space on which $G$ acts linearly has been investigated by Chevalley $[\mathbf{C h}, \mathbf{B o}]$, Kostant $[\mathbf{K o}]$, Popov $[\mathbf{P o}]$, Schwarz $[\mathbf{S c}]$, and Littelmann [Li]. In the general case, only examples have been studied, for instance by Richardson [Ri1, Ri2] or Schwarz and Wehlau [SW].

We will investigate the case where the variety $V$ is the group $G$, acting on itself by inner automorphisms. Then the subalgebra of invariant elements $C(G)=A(G)^{G}$ is the set of regular class functions. We assume in the remainder of the paper that $G$ is semisimple and simply connected. Richardson proved in [Ri1] that the following result holds under these assumptions.

Theorem 1. There exists a $G$-stable vector subspace $E$ of $A(G)$ such that the product map of $A(G)$ induces a vector space isomorphism from $C(G) \otimes_{k} E$ onto $A(G)$.

Richardson's proof is based on a study of the geometric properties of the conjugacy classes of $G$ and relies on heavy results of commutative algebra like the Quillen-Suslin theorem. Furthermore, as Richardson himself observed, his method gives only the existence of a subspace $E$, and does not tell how to choose an explicit $E$. One can ask for instance (see Sect. 12.1 in [Ri1]) if it is possible to find a subspace $E$ which behaves nicely in relation to the 
Peter-Weyl decomposition of $A(G)$, that is, the decomposition into isotypical components for the left regular representation of $G$.

The aim of this paper is to provide an alternate proof of Richardson's theorem. Our method gives a more rigid choice for $E$, which satisfies the condition stated above. It relies on canonical bases, which are a quite recent tool in representation theory. The source of this method can be traced back to a paper of Joseph and Letzter [JL], who acknowledge an idea of Polo. Our main reference for canonical bases will be Lusztig's book [Lu2], whose notations will be recalled but not explained.

\section{A graded quantized model and its canonical basis.}

In this section, tensor products and linear duals are taken over the field $\mathbb{Q}(v)$ of rational functions in one indeterminate.

2.1. Notations. We choose a maximal torus $T$ in $G$. The weight lattice $X$ is the character group of $T$. The coroot lattice $Y$ is the dual lattice of $X$, the duality pairing between $X$ and $Y$ being denoted by $\langle\rangle:, Y \times X \rightarrow \mathbb{Z}$. The choice of a Borel subgroup $B$ containing $T$ affords a set $I \subseteq Y$ of simple coroots and an injection $\left(I \rightarrow X, i \mapsto i^{\prime}\right)$ that gives the corresponding simple roots. The dominant integral weights form a cone $X^{+}$in the weight lattice. The set $I$ is a basis of the lattice $Y$. We assume that a symmetric bilinear form $\left(\nu, \nu^{\prime}\right) \mapsto \nu \cdot \nu^{\prime}$ is given on $Y$ so that $i \cdot i$ is a positive even integer and $2(i \cdot j) /(i \cdot i)=\left\langle i, j^{\prime}\right\rangle$ for all $i, j$ in $I$.

We define on $X^{+}$two order relations. For any $\nu, \nu^{\prime}$ in $X^{+}$, we say that $\nu \leq \nu^{\prime}$ whenever $\nu^{\prime}-\nu \in \sum_{i \in I} \mathbb{N} i^{\prime}$ and that $\nu \preccurlyeq \nu^{\prime}$ whenever $\nu^{\prime}-\nu \in X^{+}$. The poset $\left(X^{+}, \preccurlyeq\right)$ is a distributive lattice.

Let $v$ be an indeterminate. From the data above, one can define the $\mathbb{Q}(v)$-algebra $\mathbf{f}$, generated by the symbols $\left(\theta_{i}\right)_{i \in I}$ submitted to the quantized Serre relations ([Lu2], Chap. 1 and $\S 33.1)$. One then defines as in Chapter 3 of $[\mathbf{L u 2}]$ the quantized enveloping $\mathbb{Q}(v)$-algebra $\mathbf{U}$ and its involutive automorphism $\omega$. Following $§ \S 3.4-3.5$ in [Lu2], we denote the category of weight $\mathbf{U}$-modules by $\mathcal{C}$ and its full subcategory of integrable $\mathbf{U}$-modules by $\mathcal{C}^{\prime}$. Given a dominant integral weight $\lambda$, there is a unique simple object $\Lambda_{\lambda}$ in $\mathcal{C}^{\prime}$ with highest weight $\lambda$ and highest weight vector $\eta_{\lambda}$, and a unique simple object ${ }^{\omega} \Lambda_{\lambda}$ in $\mathcal{C}^{\prime}$ with lowest weight $-\lambda$ and lowest weight vector $\xi_{-\lambda}$ ([Lu2], §3.5). In $\S 14.4$ of [Lu2], Lusztig defines the canonical basis $\mathbf{B}$ of $\mathbf{f}$ and its family of subsets $\mathbf{B}(\lambda)$, where $\lambda \in X^{+}$. An immediate consequence of these definitions is the following fact.

Lemma 2. For any $b \in \mathbf{B}$, there is a dominant integral weight $\varepsilon(b)$ such that $\left\{\lambda \in X^{+} \mid b \in \mathbf{B}(\lambda)\right\}=\varepsilon(b)+X^{+}$.

Proof. With the notations of $\S 14.4$ of $[\mathbf{L u 2}], b$ belongs to $\mathbf{B}(\lambda)$ if and only if the inequality $\langle i, \lambda\rangle \geq \min \left\{n \mid b \in{ }^{\sigma} \mathbf{B}_{i, n}\right\}$ holds true for all $i \in I$. It is 
therefore sufficient to set $\varepsilon(b)$ so that for all $i \in I,\langle i, \varepsilon(b)\rangle=\min \{n \mid b \in$ $\left.{ }^{\sigma} \mathbf{B}_{i, n}\right\}$.

2.2. A graded quantized model for $A(G)$. By $\S 25.1$ in [Lu2], for any dominant integral weights $\lambda, \mu \in X^{+}$, there are unique maps of $\mathbf{U}$-modules $i_{\lambda, \mu}: \Lambda_{\lambda+\mu} \rightarrow \Lambda_{\lambda} \otimes \Lambda_{\mu}$ and ${ }^{\omega} i_{\lambda, \mu}:{ }^{\omega} \Lambda_{\lambda+\mu} \rightarrow{ }^{\omega} \Lambda_{\mu} \otimes{ }^{\omega} \Lambda_{\lambda}$ such that $i_{\lambda, \mu}\left(\eta_{\lambda+\mu}\right)=$ $\eta_{\lambda} \otimes \eta_{\mu}$ and ${ }^{\omega} i_{\lambda, \mu}\left(\xi_{-\lambda-\mu}\right)=\xi_{-\mu} \otimes \xi_{-\lambda}$.

Using the antipode of $\mathbf{U}$, the dual vector space $M^{*}$ of a $\mathbf{U}$-module $M$ can be viewed as a $\mathbf{U}$-module. If $M$ and $N$ are $\mathbf{U}$-modules and if one of them is finite-dimensional, then the $\mathbf{U}$-modules $(M \otimes N)^{*}$ and $N^{*} \otimes M^{*}$ are naturally isomorphic. The dual of a finite-dimensional object of $\mathcal{C}^{\prime}$ belongs to $\mathcal{C}^{\prime}$.

For any dominant integral weight $\lambda$, we define the $\mathbf{U}$-module $H^{\lambda}=\left({ }^{\omega} \Lambda_{\lambda} \otimes\right.$ $\left.\Lambda_{\lambda}\right)^{*}$. We also set $H=\bigoplus_{\lambda \in X^{+}} H^{\lambda}$. The family of maps

$$
\left(\begin{array}{rl}
H^{\lambda} \otimes H^{\mu} & \rightarrow H^{\lambda+\mu} \\
\left(\Lambda_{\lambda}\right)^{*} \otimes\left({ }^{\omega} \Lambda_{\lambda}\right)^{*} \otimes\left(\Lambda_{\mu}\right)^{*} \otimes\left({ }^{\omega} \Lambda_{\mu}\right)^{*} & \rightarrow\left(\Lambda_{\lambda+\mu}\right)^{*} \otimes\left({ }^{\omega} \Lambda_{\lambda+\mu}\right)^{*} \\
p \otimes q \otimes r \otimes s & \mapsto\left(i_{\lambda, \mu}\right)^{*}(r \otimes p) \otimes\left({ }^{\omega} i_{\lambda, \mu}\right)^{*}(q \otimes s)
\end{array}\right)
$$

induces a product $m: H \otimes H \rightarrow H$ which endows $H$ with the structure of a $X^{+}$-graded algebra. One can easily show that this algebra is associative and has a unit.

By Proposition 25.1.4 (a) in [Lu2], for any dominant integral weight $\lambda$ there is a unique $\mathbf{U}$-linear map $\delta_{\lambda}:{ }^{\omega} \Lambda_{\lambda} \otimes \Lambda_{\lambda} \rightarrow \mathbb{Q}(v)$ such that $\delta_{\lambda}\left(\xi_{-\lambda} \otimes \eta_{\lambda}\right)=$ 1 , where $\mathbb{Q}(v)$ is considered as a $\mathbf{U}$-module via the co-unit of $\mathbf{U}$. This form $\delta_{\lambda}$ is a $\mathbf{U}$-invariant element in $H^{\lambda}$.

For any two dominant integral weights $\lambda$ and $\mu$, Lusztig defines in $\S 25.1 .5$ of $[\mathbf{L u 2}]$ the map $t_{\lambda}:{ }^{\omega} \Lambda_{\lambda+\mu} \otimes \Lambda_{\lambda+\mu} \rightarrow{ }^{\omega} \Lambda_{\mu} \otimes \Lambda_{\mu}$ as the composition

$$
\begin{aligned}
& { }^{\omega} \Lambda_{\lambda+\mu} \otimes \Lambda_{\lambda+\mu} \stackrel{{ }^{\omega} i_{\lambda, \mu} \otimes i_{\lambda, \mu}}{\longrightarrow}{ }^{\omega} \Lambda_{\mu} \otimes{ }^{\omega} \Lambda_{\lambda} \otimes \Lambda_{\lambda} \otimes \Lambda_{\mu} \\
& \stackrel{\mathrm{id} \otimes \delta_{\lambda} \otimes \mathrm{id}}{\longrightarrow}{ }^{\omega} \Lambda_{\mu} \otimes \mathbb{Q}(v) \otimes \Lambda_{\mu} .
\end{aligned}
$$

\section{Lemma 3.}

(a) The dual map $\left(t_{\lambda}\right)^{*}: H^{\mu} \rightarrow H^{\lambda+\mu}$ is injective and coincides with the left multiplication by $\delta_{\lambda}$ in the algebra $H$.

(b) In the algebra $H$, one has $\delta_{\lambda} \delta_{\mu}=\delta_{\lambda+\mu}$ for any dominant integral weights $\lambda$ and $\mu$.

Proof. The injectivity of $\left(t_{\lambda}\right)^{*}$ follows from the surjectivity of $t_{\lambda}$, which is shown in [Lu2], Lemma 25.1.6 (c). Let us write $\delta_{\lambda}=\sum_{i} p_{i} \otimes q_{i}$ in $\left(\Lambda_{\lambda}\right)^{*} \otimes$ $\left({ }^{\omega} \Lambda_{\lambda}\right)^{*}$. Then for any elements $\sum_{j} r_{j} \otimes s_{j} \in\left(\Lambda_{\mu}\right)^{*} \otimes\left({ }^{\omega} \Lambda_{\mu}\right)^{*}$ and $\sum_{k} t_{k} \otimes u_{k} \in$ 
${ }^{\omega} \Lambda_{\lambda+\mu} \otimes \Lambda_{\lambda+\mu}$, we have

$$
\begin{aligned}
& \left\langle\delta_{\lambda} \times\left(\sum_{j} r_{j} \otimes s_{j}\right), \sum_{k} t_{k} \otimes u_{k}\right\rangle \\
& =\sum_{i, j, k}\left\langle\left(i_{\lambda, \mu}\right)^{*}\left(r_{j} \otimes p_{i}\right) \otimes\left({ }^{\omega} i_{\lambda, \mu}\right)^{*}\left(q_{i} \otimes s_{j}\right), t_{k} \otimes u_{k}\right\rangle \\
& =\sum_{i, j, k}\left\langle r_{j} \otimes p_{i} \otimes q_{i} \otimes s_{j},{ }^{\omega} i_{\lambda, \mu}\left(t_{k}\right) \otimes i_{\lambda, \mu}\left(u_{k}\right)\right\rangle \\
& =\sum_{j, k}\left\langle r_{j} \otimes \delta_{\lambda} \otimes s_{j},\left({ }^{\omega} i_{\lambda, \mu} \otimes i_{\lambda, \mu}\right)\left(t_{k} \otimes u_{k}\right)\right\rangle \\
& =\left\langle\sum_{j} r_{j} \otimes s_{j}, t_{\lambda}\left(\sum_{k} t_{k} \otimes u_{k}\right)\right\rangle .
\end{aligned}
$$

This calculation proves (a).

Now the linear form $\delta_{\lambda} \delta_{\mu}$ on ${ }^{\omega} \Lambda_{\lambda+\mu} \otimes \Lambda_{\lambda+\mu}$ is $\mathbf{U}$-linear and takes the value 1 on the element $\xi_{-\lambda-\mu} \otimes \eta_{\lambda+\mu}$, since it can be written as $\left(t_{\lambda}\right)^{*}\left(\delta_{\mu}\right)=$ $\delta_{\mu} \circ t_{\lambda}$. Therefore it coincides with $\delta_{\lambda+\mu}$, which proves (b).

2.3. Dual-based modules and isotypical decompositions. The simple objects of the category $\mathcal{C}^{\prime}$ are the $\mathbf{U}$-modules $\Lambda_{\sigma}$, where $\sigma$ is a dominant integral weight; they are pairwise non-isomorphic. Given an object $M$ in $\mathcal{C}^{\prime}$ and a dominant integral weight $\sigma$, we denote the sum of the simple subobjects of $M$ isomorphic to $\Lambda_{\sigma}$ by $M[\sigma]$. By complete reducibility, we have $M=\bigoplus_{\sigma \in X^{+}} M[\sigma]$. Given $P \subseteq X^{+}$, we denote the subspace $\bigoplus_{\sigma \in P} M[\sigma]$ by $M[P]$. For short, we will write $\geq \sigma$ instead of $\left\{\tau \in X^{+} \mid \tau \geq \sigma\right\}, \nless \sigma$ instead of $\left\{\tau \in X^{+} \mid \tau \nless \sigma\right\}$, and so on.

In Chapter 27 of his book [Lu2], Lusztig defines the notion of a based module. A based module is a pair $(M, B)$ consisting of a finite-dimensional U-module $M$ which belongs to $\mathcal{C}^{\prime}$ and a $\mathbb{Q}(v)$-basis $B$ of $M$ satisfying several properties stated in $\S 27.1 .2$ of [Lu2]. Based modules are the objects of a category: A morphism from the based module $(M, B)$ to the based module $\left(M^{\prime}, B^{\prime}\right)$ is a morphism $f: M \rightarrow M^{\prime}$ of $\mathbf{U}$-modules such that $f(B) \subseteq B^{\prime} \cup\{0\}$ and such that the set $B \cap \operatorname{ker} f$ is a basis of ker $f$.

We define a dual-based module as a pair $(M, B)$ consisting of a finitedimensional U-module $M$ which belongs to $\mathcal{C}^{\prime}$ and a $\mathbb{Q}(v)$-basis $B$ of $M$ such that the dual module $M^{*}$ together with the basis $B^{*}$ dual to $B$ is a based module. Dual-based modules form a category, the morphisms between two dual-based modules being defined in the same way as morphisms between based modules.

For any dual-based module $(M, B)$ and any dominant integral weight $\sigma$, we put

$$
B[\sigma]=(B \cap M[\leq \sigma]) \backslash(B \cap M[<\sigma]) .
$$

The following properties of dual-based modules are direct consequences of similar properties of based modules. 
Proposition 4. Let $(M, B)$ be a dual-based module and let $\sigma$ be a dominant integral weight.

(a) The subspaces $M[\leq \sigma]$ and $M[<\sigma]$ are spanned over $\mathbb{Q}(v)$ by their intersection with $B$.

(b) The restriction of the canonical surjection $p: M[\leq \sigma] \rightarrow M[\leq \sigma] / M[<$ $\sigma]$ to $B[\sigma]$ is injective and the pair $(M[\leq \sigma] / M[<\sigma], p(B[\sigma]))$ is a dual-based module.

(c) When $\sigma$ runs over $X^{+}$, the sets $B[\sigma]$ form a partition of $B$.

(d) Let $\left(M^{\prime}, B^{\prime}\right)$ be a sub-dual-based module of $(M, B)$ and assume that $M$ has only one nonzero isotypical component. Then the $\mathbb{Q}(v)$-vector space $M^{\prime \prime}$ spanned by $B \backslash B^{\prime}$ is a complementary sub-U-module of $M^{\prime}$ in $M$ and the pair $\left(M^{\prime \prime}, B \backslash B^{\prime}\right)$ is a dual-based module.

(e) Let $\left(M^{\prime}, B^{\prime}\right)$ be a dual-based module and assume that $\mathbf{U}$ acts trivially on $M$ or on $M^{\prime}$. Then $\left(M \otimes M^{\prime}, B \otimes B^{\prime}\right)$ is a dual-based module, where $B \otimes B^{\prime}$ denotes the set $\left\{b \otimes b^{\prime} \mid b \in B, b^{\prime} \in B^{\prime}\right\}$.

Proof. Proposition 27.1.8 in [Lu2] asserts that for any dominant integral weight $\tau$ and any based module $(N, C)$, the submodule $N[\geq \tau]$ is spanned over $\mathbb{Q}(v)$ by its intersection with $C$. One deduces from this fact that the submodule $N[P]$ is spanned over $\mathbb{Q}(v)$ by its intersection with $C$ for any subset $P \subseteq X^{+}$such that $P+\left(\sum_{i} \mathbb{N} i^{\prime}\right) \subseteq P$. In particular, this property holds for $N\left[\not \subset \sigma^{*}\right]$ and $N\left[\nless \sigma^{*}\right]$, where $\sigma^{*}$ is the highest weight of $\left(\Lambda_{\sigma}\right)^{*}$. Applying this result to the case of the based module $\left(M^{*}, B^{*}\right)$ and taking orthogonals, we obtain Property (a).

Property (a) proves that the restriction of $p$ defines a bijection from $B[\sigma]$ onto a basis of the $\mathbb{Q}(v)$-vector space $M[\leq \sigma] / M[<\sigma]$. To check that the pair $(M[\leq \sigma] / M[<\sigma], p(B[\sigma]))$ satisfies all the axioms of a dual-based module, it suffices to use duality as in the proof of Property (a) and to refer to the definition of based modules in $\S 27.1 .2$ of $[\mathbf{L u 2}]$. Property (b) is proved.

Choose any $x$ in $B$. We can find $\sigma \in X^{+}$such that $x \in M[\leq \sigma]$ and such that $\sigma$ is minimal for this property with respect to the order $\leq$. Since $B$ is a basis of $M$, the element $x$ does not belong to the span of $\bigcup_{\tau<\sigma}(B \cap M[\leq \tau])$. By Property (a), one deduces that $x$ does not belong to $M[<\sigma]$ and therefore that $x$ belongs to $B[\sigma]$. We have proved that $B$ is the union of its subsets $B[\sigma]$, and it remains us to show that these sets $B[\sigma]$ are pairwise disjoint. Suppose that $B[\sigma]$ and $B[\tau]$ share a certain element $x$. Then $M[\leq \sigma]$ and $M[\leq \tau]$ intersect non-trivially. This implies that $\sigma-\tau$ belongs to the root lattice $\sum_{i} \mathbb{Z} i^{\prime}$, and thus there exists a weight $\rho$ less than or equal to $\sigma$ and $\tau$ such that $M[\leq \sigma] \cap M[\leq \tau]=M[\leq \rho]$. Since $x$ belongs to $M[\leq \rho]$ but not to $M[<\sigma]$, we cannot have $\rho<\sigma$. Therefore $\rho=\sigma$, and similarly $\rho=\tau$. Therefore $\sigma=\tau$, which completes the proof of Property (c). 
Finally Property (d) is a consequence of the proof of Proposition 27.1.7 in [Lu2], and Property (e) follows by dualizing the construction given in $\S 27.3$ and Theorem 27.3.2 of [Lu2].

It is of course possible to extend the notion of (dual-) based module to the case of an infinite-dimensional $\mathbf{U}$-module which is graded with finitedimensional graded components. In this case, the basis is required to be compatible with the decomposition of the module as the direct sum of its graded components.

2.4. The basis of $H$. By $\S \S 24.3$ and 27.3 .4 in [Lu2], each module ${ }^{\omega} \Lambda_{\lambda} \otimes \Lambda_{\lambda}$ has a canonical basis, with which it forms a based module. By Proposition 27.3.5 (a) in [Lu2], the map $t_{\lambda}:{ }^{\omega} \Lambda_{\lambda+\mu} \otimes \Lambda_{\lambda+\mu} \rightarrow{ }^{\omega} \Lambda_{\mu} \otimes \Lambda_{\mu}$ is a morphism of based modules.

Each module $H^{\lambda}=\left({ }^{\omega} \Lambda_{\lambda} \otimes \Lambda_{\lambda}\right)^{*}$ comes therefore with the dual basis $B_{\lambda}$, so that the pair $\left(H^{\lambda}, B_{\lambda}\right)$ is a dual-based module. By Lemma $3(\mathrm{a})$, the left multiplication by $\delta_{\lambda}$ defines an injective morphism of dual-based modules from $\left(H^{\mu}, B_{\mu}\right)$ to $\left(H^{\lambda+\mu}, B_{\lambda+\mu}\right)$.

In particular, we get an injective map from $B_{\mu}$ to $B_{\lambda+\mu}$. By Lemma 3(b) these maps form a directed system of injective maps between sets, and we denote its limit ${ }^{1}$ by $B_{\infty}=\underline{\lim } B_{\lambda}$. We denote the canonical injective map $B_{\lambda} \rightarrow B_{\infty}$ by $\iota_{\lambda}$. By Proposition 27.2.2 in [Lu2], this directed system is compatible with the decompositions $B_{\lambda}=\bigsqcup_{\sigma \in X^{+}} B_{\lambda}[\sigma]$, which yields a similar decomposition $B_{\infty}=\bigsqcup_{\sigma \in X^{+}} B_{\infty}[\sigma]$.

Lemma 5. Given $x \in B_{\infty}$, there is a dominant integral weight $\varepsilon(x)$ such that $\left\{\lambda \in X^{+} \mid x \in \iota_{\lambda}\left(B_{\lambda}\right)\right\}=\varepsilon(x)+X^{+}$.

Proof. By duality, the assertion is equivalent to the following fact: For any $\lambda, \mu, \nu \in X^{+}$such that $\lambda \preccurlyeq \nu$ and $\mu \preccurlyeq \nu$ and any $y$ in the canonical basis of ${ }^{\omega} \Lambda_{\nu} \otimes \Lambda_{\nu}$, the non-vanishing of both $t_{\lambda}(y)$ and $t_{\mu}(y)$ implies that of $t_{\sup (\lambda, \mu)}(y)$, where $\sup (\cdot, \cdot)$ is the supremum in the distributive lattice $\left(X^{+}, \preccurlyeq\right)$. In turn, this fact is a direct consequence of Proposition 25.1.10 in [Lu2] and Lemma 2.

Lemma 6. The set $B_{\lambda}[0]$ is reduced to the element $\delta_{\lambda}$.

Proof. The space $H^{\lambda}[0]=\operatorname{Hom}_{\mathbf{U}}\left({ }^{\omega} \Lambda_{\lambda} \otimes \Lambda_{\lambda}, \mathbb{Q}(v)\right)$ has dimension at most one, since ${ }^{\omega} \Lambda_{\lambda} \otimes \Lambda_{\lambda}$ is generated by a single element, namely $\xi_{-\lambda} \otimes \eta_{\lambda}$. Therefore $B_{\lambda}[0]$ has at most one element and it suffices to show that $\delta_{\lambda} \in B_{\lambda}$. We observe that the kernel of $\delta_{\lambda}$ is $\left({ }^{\omega} \Lambda_{\lambda} \otimes \Lambda_{\lambda}\right)[>0]$, which by Proposition 27.1.8 in [Lu2] is spanned over $\mathbb{Q}(v)$ by its intersection with the canonical basis of ${ }^{\omega} \Lambda_{\lambda} \otimes \Lambda_{\lambda}$. Therefore $\delta_{\lambda}$ vanishes on all elements of this canonical basis but one. The exception is the vector $\xi_{-\lambda} \otimes \eta_{\lambda}$ : It belongs to the canonical basis

\footnotetext{
${ }^{1}$ This limit $B_{\infty}$ is, in a certain sense, the basis dual to the canonical basis of the subspace $\dot{U} 1_{0}$ of Lusztig's modified quantized enveloping algebra, see Chap. 23 of [Lu2].
} 
by Theorem 24.3 .3 in [Lu2] and $\delta_{\lambda}$ evaluates to 1 on it. This shows that $\delta_{\lambda}$ belongs to the basis dual to the canonical basis of ${ }^{\omega} \Lambda_{\lambda} \otimes \Lambda_{\lambda}$, that is to say $\delta_{\lambda}$ belongs to $B_{\lambda}$.

The direct sum of the dual-based modules $\left(H^{\lambda}, B_{\lambda}\right)$ will be denoted by $(H, B)$. Lemma 6 tells that $B[0]=\left\{\delta_{\lambda} \mid \lambda \in X^{+}\right\}$and Proposition 4(a) implies that the pair $(H[0], B[0])$ is a dual-based module. By Lemma 3(a), for any $\lambda \in X^{+}$, the left multiplication by $\delta_{\lambda}$ is an injective morphism from the dual-based module $(H, B)$ into itself.

\subsection{A filtration of $H$ and the freeness theorem for its associated} graded. The dual-based module $(H, B)$ is filtered by the family of submodules $(H[\leq \sigma], B \cap H[\leq \sigma])$, the indexing set being the poset $\left(X^{+}, \leq\right)$. The associated graded dual-based module is $\bigoplus_{\sigma \in X^{+}}\left(\operatorname{gr}^{\sigma}(H), \mathcal{B}[\sigma]\right)$, where $\operatorname{gr}^{\sigma}(H)=H[\leq \sigma] / H[<\sigma]$ and $\mathcal{B}[\sigma]$ is the image of $B[\sigma]=\bigsqcup_{\lambda \in X^{+}} B_{\lambda}[\sigma]$ under the canonical surjection $p: H[\leq \sigma] \rightarrow \operatorname{gr}^{\sigma}(H)$.

We view $H$ as the regular left $H$-module. The subspace $H[0]$ acts by morphisms of $\mathbf{U}$-modules; therefore its action stabilizes each isotypical component of $H$ and induces an action on any $\operatorname{gr}^{\sigma}(H)$.

We now fix a dominant integral weight $\sigma$. We define

$$
B[\sigma]^{\operatorname{prim}}=\left\{\iota_{\varepsilon(x)}^{-1}(x) \mid x \in B_{\infty}[\sigma]\right\}=\bigsqcup_{\lambda \in X^{+}}\left\{x \in B_{\lambda}[\sigma] \mid \varepsilon\left(\iota_{\lambda}(x)\right)=\lambda\right\},
$$

and we call $\mathcal{B}[\sigma]^{\text {prim }}$ its image under the canonical surjection $p$. We denote by $K^{\sigma}$ the $\mathbb{Q}(v)$-vector subspace spanned in $\operatorname{gr}^{\sigma}(H)$ by $\mathcal{B}[\sigma]^{\text {prim }}$.

\section{Proposition 7.}

(a) The action of $\delta_{\lambda}$ on $\operatorname{gr}^{\sigma}(H)$ induces an injective morphism from the dual-based module $\left(\mathrm{gr}^{\sigma}(H), \mathcal{B}[\sigma]\right)$ into itself.

(b) The family of sets $\left(\delta_{\lambda} \cdot \mathcal{B}[\sigma]^{\text {prim }}\right)_{\lambda \in X^{+}}$form a partition of $\mathcal{B}[\sigma]$.

(c) The pair $\left(K^{\sigma}, \mathcal{B}[\sigma]^{\text {prim }}\right)$ is a dual-based module.

Proof. Assertion (a) follows from the fact that the left multiplication by $\delta_{\lambda}$ is an injective morphism from the dual-based module $(H, B)$ into itself.

As for Assertion (b), we consider an element $x \in B_{\mu}[\sigma]$. Let $\nu=\varepsilon\left(\iota_{\mu}(x)\right)$. By Lemma $5, \lambda=\mu-\nu$ belongs to $X^{+}$and there exists $y \in B_{\nu}[\sigma]$ such that $\iota_{\nu}(y)=\iota_{\mu}(x)$. By construction, $y \in B[\sigma]^{\text {prim }}$ and $p(x)$ is the image of $p(y)$ under the action of $\delta_{\lambda}$. This proves that $\mathcal{B}[\sigma]=\bigcup_{\lambda \in X^{+}}\left(\delta_{\lambda} \cdot \mathcal{B}[\sigma]^{\text {prim }}\right)$. A similar reasoning based on Lemma 5 and on Assertion (a) shows that the union is disjoint.

To prove Assertion (c), it is enough to show that for all dominant integral weight $\lambda$, the pair $\left(K^{\sigma} \cap \operatorname{gr}^{\sigma}\left(H^{\lambda}\right), \mathcal{B}[\sigma]^{\operatorname{prim}} \cap \operatorname{gr}^{\sigma}\left(H^{\lambda}\right)\right)$ is a dual-based module. This is trivial for $\lambda=0$. The case of a general $\lambda$ will be proved by induction on $\sum_{i}\langle i, \lambda\rangle$. Assume that $\lambda \neq 0$ is given. By the induction hypothesis, we can assume that the pair $\left(K^{\sigma} \cap \operatorname{gr}^{\sigma}\left(H^{\mu}\right), \mathcal{B}[\sigma]^{\text {prim }} \cap \operatorname{gr}^{\sigma}\left(H^{\mu}\right)\right)$ is a dual-based 
module for all $\mu \in X^{+}$such that $\mu \prec \lambda$. Assertion (b) then says that the pair

$$
\left(\bigoplus_{\mu \in X^{+}, \mu \prec \lambda} \delta_{\lambda-\mu} \cdot\left(K^{\sigma} \cap \operatorname{gr}^{\sigma}\left(H^{\mu}\right)\right), \bigsqcup_{\mu \in X^{+}, \mu \prec \lambda} \delta_{\lambda-\mu} \cdot\left(\mathcal{B}[\sigma]^{\operatorname{prim}} \cap \operatorname{gr}^{\sigma}\left(H^{\mu}\right)\right)\right)
$$

is a sub-dual-based module of $\left(\operatorname{gr}^{\sigma}\left(H^{\lambda}\right), \mathcal{B}[\sigma] \cap \operatorname{gr}^{\sigma}\left(H^{\lambda}\right)\right)$ and that

$$
\begin{aligned}
\mathcal{B}[\sigma]^{\operatorname{prim}} & \cap \operatorname{gr}^{\sigma}\left(H^{\lambda}\right) \\
= & \left(\mathcal{B}[\sigma] \cap \operatorname{gr}^{\sigma}\left(H^{\lambda}\right)\right) \backslash\left(\bigsqcup_{\mu \in X^{+}, \mu \prec \lambda} \delta_{\lambda-\mu} \cdot\left(\mathcal{B}[\sigma]^{\operatorname{prim}} \cap \operatorname{gr}^{\sigma}\left(H^{\mu}\right)\right)\right) .
\end{aligned}
$$

Now Assertion (c) follows from Proposition 4(d).

We now have three dual-based modules $\left(\operatorname{gr}^{\sigma}(H), \mathcal{B}[\sigma]\right),(H[0], B[0])$, and $\left(K^{\sigma}, \mathcal{B}[\sigma]^{\text {prim }}\right)$. By Proposition $4(\mathrm{e})$, the pair $\left(H[0] \otimes K^{\sigma}, B[0] \otimes \mathcal{B}[\sigma]^{\text {prim }}\right)$ is a dual-based module.

Theorem 8. The action of $H[0]$ on $\operatorname{gr}^{\sigma}(H)$ gives rise to an isomorphism from $\left(H[0] \otimes K^{\sigma}, B[0] \otimes \mathcal{B}[\sigma]^{\text {prim }}\right)$ onto $\left(\operatorname{gr}^{\sigma}(H), \mathcal{B}[\sigma]\right)$.

Proof. Since $\mathbf{U}$ acts trivially on $H[0]$, the U-linear action of $H[0]$ on $\operatorname{gr}^{\sigma}(H)$ induces a morphism of $\mathbf{U}$-modules from $H[0] \otimes \mathrm{gr}^{\sigma}(H)$ to $\mathrm{gr}^{\sigma}(H)$. By Proposition 7 (a) and (b), this morphism restricts to a bijection from $B[0] \otimes \mathcal{B}[\sigma]^{\text {prim }}$ onto $\mathcal{B}[\sigma]$. The theorem follows.

\section{Specialization to the classical case.}

3.1. Specialization of $\mathbf{U}$-modules. Let $\mathcal{A}$ be the $\operatorname{ring} \mathbb{Z}\left[v, v^{-1}\right]$. The field $k$ is an $\mathcal{A}$-algebra on which $v$ acts as the identity. For any $\mathcal{A}$-module ${ }_{\mathcal{A}} T$, we denote by ${ }_{k} T$ the $k$-module $k \otimes_{\mathcal{A}}{ }_{\mathcal{A}} T$ obtained by base ring change.

We call $\mathfrak{g}$ the Lie algebra of the group $G$ and we choose Chevalley generators $E_{1}, \ldots, E_{\ell}, F_{1}, \ldots, F_{\ell}, H_{1}, \ldots, H_{\ell}$ in it.

In $\S 3.1 .13$ of [Lu2] (see also Theorem 4.5 in [Lu1]), Lusztig defines an $\mathcal{A}$-form ${ }_{\mathcal{A}} \mathbf{U}$ of $\mathbf{U}$. Formulas in $\S \S 3.1 .5$ and 3.3 .3 of $[\mathbf{L u 2}]$ show that ${ }_{\mathcal{A}} \mathbf{U}$ inherits from $\mathbf{U}$ the structure of a Hopf algebra over $\mathcal{A}$. Therefore ${ }_{k} \mathbf{U}$ is a Hopf algebra over $k$. Furthermore, since the quantized Serre relations are verified by the simple root vectors in ${ }_{\mathcal{A}} \mathbf{U}$, there is a natural morphism of Hopf algebras $c: U(\mathfrak{g}) \rightarrow{ }_{k} \mathbf{U}$. Thanks to $c$, every ${ }_{k} \mathbf{U}$-module has a natural structure of a $U(\mathfrak{g})$-module.

We use the standard strategy to specialize a finite-dimensional U-module $M$ : We first choose a $\mathbb{Q}(v)$-basis $B$ of $M$ such that the $\mathcal{A}$-submodule ${ }_{\mathcal{A}} M$ spanned by $B$ in $M$ is stable under the action of ${ }_{\mathcal{A}} \mathbf{U}$, and then ${ }_{k} M$ is a $U(\mathfrak{g})$ module. So what we really specialize is the pair $(M, B)$. Thanks to Condition (b) in Definition 27.1.2 of [Lu2], based modules satisfy the required 
condition to be specializable. One can also construct new specializable pairs by standard procedures like dualization, tensor product, or twisting with $\omega$, and then the specialization commutes with these constructions. We extend this framework to infinite-dimensional $\mathbf{U}$-modules provided that they are graded with finite-dimensional graded components and that their bases consist of homogeneous elements.

Let $\lambda \in X^{+}$. In Theorem 14.4.11 of [Lu2], Lusztig constructs a $\mathbb{Q}(v)$-basis $\mathbf{B}\left(\Lambda_{\lambda}\right)$ of $\Lambda_{\lambda}$ so that $\left(\Lambda_{\lambda}, \mathbf{B}\left(\Lambda_{\lambda}\right)\right)$ is a based module. Lusztig shows in $\S 33.1 .2$ of $[\mathbf{L u 2}]$ that the specialized module ${ }_{k}\left(\Lambda_{\lambda}\right)$ is a simple highest weight module with highest weight $\lambda$. The basis $\mathbf{B}\left(\Lambda_{\lambda}\right)$ endows ${ }_{k}\left(\Lambda_{\lambda}\right)$ with a preferred highest weight vector ${ }_{k} \eta_{\lambda}$. Take another $\mu \in X^{+}$. By Proposition 25.1.2 in [Lu2], the map $i_{\lambda, \mu}: \Lambda_{\lambda+\mu} \rightarrow \Lambda_{\lambda} \otimes_{\mathbb{Q}(v)} \Lambda_{\mu}$ sends the $\mathcal{A}$-submodule spanned by $\mathbf{B}\left(\Lambda_{\lambda+\mu}\right)$ in $\Lambda_{\lambda+\mu}$ into the $\mathcal{A}$-submodule spanned by $\mathbf{B}\left(\Lambda_{\lambda}\right) \otimes \mathbf{B}\left(\Lambda_{\mu}\right)$ in $\Lambda_{\lambda} \otimes_{\mathbb{Q}(v)} \Lambda_{\mu}$. It therefore specializes to the morphism of $U(\mathfrak{g})$-modules ${ }_{k}\left(i_{\lambda, \mu}\right):{ }_{k}\left(\Lambda_{\lambda+\mu}\right) \rightarrow{ }_{k}\left(\Lambda_{\lambda}\right) \otimes_{k}{ }_{k}\left(\Lambda_{\mu}\right)$ that sends ${ }_{k} \eta_{\lambda+\mu}$ to ${ }_{k} \eta_{\lambda} \otimes_{k} \eta_{\mu}$.

Similarly, the $\mathbf{U}$-module ${ }^{\omega} \Lambda_{\lambda}$ comes with a canonical basis ${ }^{\omega} \mathbf{B}\left(\Lambda_{\lambda}\right)$. Therefore it can be specialized to the $U(\mathfrak{g})$-module ${ }_{k}\left({ }^{\omega} \Lambda_{\lambda}\right)$, which is a simple lowest weight module with lowest weight $-\lambda$ and lowest weight vector ${ }_{k} \xi_{-\lambda}$. The specialization of ${ }^{\omega} i_{\lambda, \mu}:{ }^{\omega} \Lambda_{\lambda+\mu} \rightarrow{ }^{\omega} \Lambda_{\mu} \otimes_{\mathbb{Q}(v)}{ }^{\omega} \Lambda_{\lambda}$ is the morphism of $U(\mathfrak{g})$ modules ${ }_{k}\left({ }^{\omega} i_{\lambda, \mu}\right):{ }_{k}\left({ }^{\omega} \Lambda_{\lambda+\mu}\right) \rightarrow{ }_{k}\left({ }^{\omega} \Lambda_{\mu}\right) \otimes_{k}{ }_{k}\left({ }^{\omega} \Lambda_{\lambda}\right)$ that sends ${ }_{k} \xi_{-\lambda-\mu}$ to ${ }_{k} \xi_{-\mu} \otimes{ }_{k} \xi_{-\lambda}$.

The family $\left({ }_{k}\left(\Lambda_{\sigma}\right)\right)_{\sigma \in X^{+}}$affords a complete set of pairwise non-isomorphic finite-dimensional simple $U(\mathfrak{g})$-modules. Given a finite-dimensional $U(\mathfrak{g})$ module $M$ and a dominant integral weight $\sigma$, we denote its isotypical component of type ${ }_{k}\left(\Lambda_{\sigma}\right)$ by $M[\sigma]$. Given $P \subseteq X^{+}$, we denote the subspace $\bigoplus_{\sigma \in P} M[\sigma]$ by $M[P]$.

Proposition 9. Let $(M, B)$ be a dual-based module and ${ }_{k} M$ its specialization. Then for any $\sigma \in X^{+}$, the dual-based modules $(M[\leq \sigma], B \cap M[\leq \sigma])$ and $(M[<\sigma], B \cap M[<\sigma])$ specialize to $\left({ }_{k} M\right)[\leq \sigma]$ and $\left({ }_{k} M\right)[<\sigma]$, respectively. In particular $(M[0], B \cap M[0])$ specializes to $\left({ }_{k} M\right)[0]$.

Proof. We will only prove the case of $(M[\leq \sigma], B \cap M[\leq \sigma])$. One can enumerate the weights in $\leq \sigma$ as a finite sequence $\tau_{1}, \ldots, \tau_{n}$ such that $\tau_{i} \leq \tau_{j} \Rightarrow i \leq j$. The dual-based module $(M[\leq \sigma], B \cap M[\leq \sigma])$ is then filtered by the composition series $\left(M\left[\left\{\tau_{1}, \ldots, \tau_{i}\right\}\right], B \cap M\left[\left\{\tau_{1}, \ldots, \tau_{i}\right\}\right]\right)_{0 \leq i \leq n}$. As $\mathbf{U}$-modules, the quotient modules are isotypical of type $\Lambda_{\tau_{i}}$ and specialize therefore to isotypical modules of type ${ }_{k}\left(\Lambda_{\tau_{i}}\right)$, by the dual version of Proposition 27.1.7 in [Lu2]. Thus the specialization of $(M[\leq \sigma], B \cap M[\leq \sigma])$ has a filtration with quotients isomorphic to ${ }_{k}\left(\Lambda_{\tau_{1}}\right), \ldots$, or ${ }_{k}\left(\Lambda_{\tau_{n}}\right)$, which shows that ${ }_{k}(M[\leq \sigma]) \subseteq\left({ }_{k} M\right)[\leq \sigma]$. A similar reasoning shows that the specialization of $M / M[\leq \sigma]$ has a filtration with quotients isomorphic to modules 
of the form ${ }_{k}\left(\Lambda_{\tau}\right)$ with $\tau \not \leq \sigma$, whence

$$
\left(\left({ }_{k} M\right) /{ }_{k}(M[\leq \sigma])\right)[\leq \sigma]=\left({ }_{k}(M / M[\leq \sigma])\right)[\leq \sigma]=0 .
$$

Therefore the equality ${ }_{k}(M[\leq \sigma])=\left({ }_{k} M\right)[\leq \sigma]$ holds.

3.2. Specialization of $H$. We are now in a position where we can specialize the U-module $H$, the multiplication map $m: H \otimes_{\mathbb{Q}(v)} H \rightarrow H$, and the freeness result from Theorem 8 .

We first observe that by Theorem 24.3.3 in [Lu2], the $\mathcal{A}$-lattice spanned in $H^{\lambda}$ by the basis $B_{\lambda}$ is the same as the $\mathcal{A}$-lattice spanned by the basis dual to the basis ${ }^{\omega} \mathbf{B}\left(\Lambda_{\lambda}\right) \otimes \mathbf{B}\left(\Lambda_{\lambda}\right)$ of ${ }^{\omega} \Lambda_{\lambda} \otimes_{\mathbb{Q}(v)} \Lambda_{\lambda}$. Therefore the multiplication map $m$ sends the $\mathcal{A}$-submodule spanned in $H \otimes_{\mathbb{Q}(v)} H$ by $B \otimes B$ into the $\mathcal{A}$-submodule spanned in $H$ by $B$. It gives rise to a multiplication map ${ }_{k} m:{ }_{k} H \otimes_{k}{ }_{k} H \rightarrow{ }_{k} H$.

Proposition 10. The specialization ${ }_{k} H$ is the $U(\mathfrak{g})$-module

$$
\bigoplus_{\lambda \in X^{+}}\left({ }_{k}\left(\Lambda_{\lambda}\right)^{*} \otimes_{k k}\left({ }^{\omega} \Lambda_{\lambda}\right)^{*}\right) .
$$

The multiplication map ${ }_{k} m$ is given by Formula (1) in which the maps $\left(i_{\lambda, \mu}\right)^{*}$ and $\left({ }^{\omega} i_{\lambda, \mu}\right)^{*}$ are replaced by their specializations ${ }_{k}\left(i_{\lambda, \mu}\right)^{*}$ and ${ }_{k}\left({ }^{\omega} i_{\lambda, \mu}\right)^{*}$.

We now fix a dominant integral weight $\sigma$. By Proposition 9, the isotypical component $\left({ }_{k} H\right)[\sigma]$ is naturally isomorphic to the specialization of the dualbased module $\left(\operatorname{gr}^{\sigma}(H), \mathcal{B}[\sigma]\right)$. The specialization ${ }_{k}\left(K^{\sigma}\right)$ of $\left(K^{\sigma}, \mathcal{B}[\sigma]^{\text {prim }}\right)$ is then seen as a $U(\mathfrak{g})$-submodule of $\left({ }_{k} H\right)[\sigma]$. By Theorem 8 and Proposition 9 , we get the following result.

Theorem 11. The map ${ }_{k} m$ induces an isomorphism of $U(\mathfrak{g})$-modules from $\left({ }_{k} H\right)[0] \otimes_{k} k_{k}\left(K^{\sigma}\right)$ onto $\left({ }_{k} H\right)[\sigma]$.

3.3. The Cartan filtration on $A(G)$. To complete the proof of Theorem 1 , it only remains to relate the specialized algebra ${ }_{k} H$ to the algebra $A(G)$. We first describe this latter.

Let $M$ be a rational $G$-module. Then for any $v \in M$ and $f \in M^{*}$, the function on $G$

$$
c_{f, v}^{M}: g \mapsto\langle f, g \cdot v\rangle
$$

is regular. The map from $M^{*} \otimes_{k} M$ to $A(G)$ which sends $f \otimes v$ to $c_{f, v}^{M}$ is a morphism of $G$-modules; it is injective if $M$ is simple. By definition, its image is the coefficient space $C(M)$ of the module $M$. Then the Peter-Weyl decomposition

$$
A(G)=\bigoplus_{\lambda \in X^{+}} C\left({ }_{k}\left(\Lambda_{\lambda}\right)\right)
$$


holds. The filtration of $A(G)$ indexed by the poset $\left(X^{+}, \leq\right)$and given by the submodules

$$
A_{\lambda}(G)=\bigoplus_{\mu \in X^{+}, \mu \leq \lambda} C\left({ }_{k}\left(\Lambda_{\mu}\right)\right)
$$

is a filtration of algebra. The associated graded is

$$
\operatorname{gr}(A(G))=\bigoplus_{\lambda \in X^{+}} \operatorname{gr}^{\lambda}(A(G))
$$

where

$$
\operatorname{gr}^{\lambda}(A(G))=A_{\lambda}(G) / \sum_{\mu<\lambda} A_{\mu}(G) \simeq C\left({ }_{k}\left(\Lambda_{\lambda}\right)\right) \simeq{ }_{k}\left(\Lambda_{\lambda}\right)^{*} \otimes_{k}{ }_{k}\left(\Lambda_{\lambda}\right) .
$$

For any $\lambda, \mu \in X^{+}$, there is a unique morphism $p_{\lambda, \mu}:{ }_{k}\left(\Lambda_{\lambda}\right) \otimes_{k}{ }_{k}\left(\Lambda_{\mu}\right) \rightarrow$ ${ }_{k}\left(\Lambda_{\lambda+\mu}\right)$ of $U(\mathfrak{g})$-modules such that the composition $p_{\lambda, \mu} \circ{ }_{k}\left(i_{\lambda, \mu}\right)$ is the identity of ${ }_{k}\left(\Lambda_{\lambda+\mu}\right)$. Then the multiplication of the algebra $\operatorname{gr}(A(G))$ is defined by the family of maps

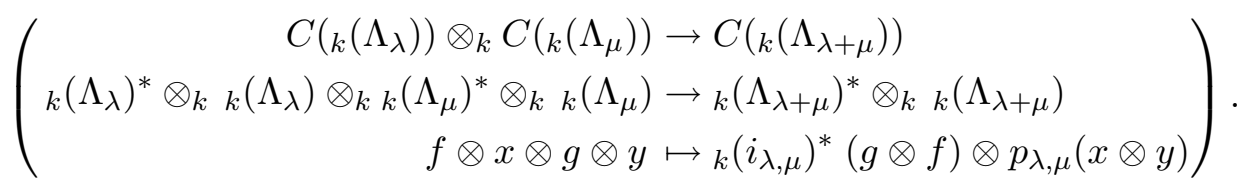

For any $\lambda \in X^{+}$, the $U(\mathfrak{g})$-module ${ }_{k}\left({ }^{\omega} \Lambda_{\lambda}\right)$ is simple with lowest weight $-\lambda$ and lowest weight vector ${ }_{k} \xi_{-\lambda}$, therefore there is a unique isomorphism $h_{\lambda}:{ }_{k}\left(\Lambda_{\lambda}\right) \rightarrow{ }_{k}\left({ }^{\omega} \Lambda_{\lambda}\right)^{*}$ of $U(\mathfrak{g})$-modules such that $\left\langle h_{\lambda}\left({ }_{k} \eta_{\lambda}\right),{ }_{k} \xi_{-\lambda}\right\rangle=1$.

Lemma 12. For any $\lambda, \mu \in X^{+}$, the relation ${ }_{k}\left({ }^{\omega} i_{\lambda, \mu}\right)^{*} \circ\left(h_{\lambda} \otimes_{k} h_{\mu}\right)=h_{\lambda+\mu} \circ$ $p_{\lambda, \mu}$ holds.

Proof. Both members of the equality to be proved are $U(\mathfrak{g})$-linear maps from $_{k}\left(\Lambda_{\lambda}\right) \otimes_{k k}\left(\Lambda_{\mu}\right)$ to ${ }_{k}\left({ }^{\omega} \Lambda_{\lambda+\mu}\right)^{*} \simeq{ }_{k}\left(\Lambda_{\lambda+\mu}\right)$. Since the latter is simple and has multiplicity one in the former, both members are equal up to a scalar. To complete the proof, it therefore suffices to check that both linear forms $\left(h_{\lambda+\mu} \circ p_{\lambda, \mu}\right)\left({ }_{k} \eta_{\lambda} \otimes{ }_{k} \eta_{\mu}\right)$ and $\left[{ }_{k}\left({ }^{\omega} i_{\lambda, \mu}\right)^{*} \circ\left(h_{\lambda} \otimes_{k} h_{\mu}\right)\right]\left({ }_{k} \eta_{\lambda} \otimes{ }_{k} \eta_{\mu}\right)$ take the value 1 when evaluated on the vector ${ }_{k} \xi_{-\lambda-\mu}$.

Let $\varphi$ be the map from $\operatorname{gr}(A(G))$ to ${ }_{k} H$ defined by the family of maps

$$
\left(\begin{array}{rl}
\operatorname{gr}^{\lambda}(A(G)) & \rightarrow{ }_{k}\left(H^{\lambda}\right) \\
{ }_{k}\left(\Lambda_{\lambda}\right)^{*} \otimes_{k}\left(\Lambda_{\lambda}\right) & \rightarrow{ }_{k}\left(\Lambda_{\lambda}\right)^{*} \otimes_{k}{ }_{k}\left({ }^{\omega} \Lambda_{\lambda}\right)^{*} \\
f \otimes x & \mapsto f \otimes h_{\lambda}(x)
\end{array}\right) .
$$

Lemma 12 implies directly the following statement.

Proposition 13. The map $\varphi$ is a $U(\mathfrak{g})$-linear isomorphism of algebras. 
Theorem 11 therefore translates immediately to a similar statement for $\operatorname{gr}(A(G))$. Since the $U(\mathfrak{g})$-module $A(G)$ is not only filtered but also graded, we can lift the submodule $\bigoplus_{\sigma \in X^{+}} \varphi^{-1}\left({ }_{k}\left(K^{\sigma}\right)\right)$ of $\operatorname{gr}(A(G))$ to a submodule $E$ of $A(G)$. Then the multiplication map in $A(G)$ restricts to an isomorphism of vector spaces from $C(G) \otimes_{k} E$ onto $A(G)$, since the graded counterpart of this restriction

$$
\operatorname{gr}(A(G))[0] \otimes_{k}\left(\bigoplus_{\sigma \in X^{+}} \varphi^{-1}\left({ }_{k}\left(K^{\sigma}\right)\right)\right) \rightarrow \bigoplus_{\sigma \in X^{+}} \operatorname{gr}(A(G))[\sigma]
$$

is itself bijective. This concludes the proof of Theorem 1 .

Remark. The author does not understand the relation between the point of view presented in this paper and the extension by Donkin [Do] of Richardson's work to the case where the ground field has a positive characteristic.

Acknowledgements. The author has the pleasure to thank N. Reshetikhin for a three-months long invitation at the University of California at Berkeley.

\section{References}

[Bo] N. Bourbaki, Groupes et Algèbres de Lie, Chapitres 4, 5 et 6, Masson, Paris, 1981, MR 39 \#1590, Zbl 0186.33001 and Zbl 0483.22001.

[Ch] C. Chevalley, Invariants of finite groups generated by reflections, Amer. J. Math., 77 (1955), 778-782, MR 17,345d, Zbl 0065.26103.

[Do] S. Donkin, On conjugating representations and adjoint representations of semisimple groups, Invent. Math., 91 (1988), 137-145, MR 89a:20047, Zbl 0639.20021.

[JL] A. Joseph and G. Letzter, Separation of variables for quantized enveloping algebras, Amer. J. Math., 116 (1994), 127-177, MR 95e:17017, Zbl 0811.17007.

[Ko] B. Kostant, Lie group representations on polynomial rings, Amer. J. Math., 85 (1963), 327-404, MR 28 \#1252, Zbl 0124.26802.

[Li] P. Littelmann, Koreguläre und äquidimensionale darstellungen, J. Algebra, 123 (1989), 193-222, MR 90e:20039, Zbl 0688.14042.

[Lu1] G. Lusztig, Finite dimensional Hopf algebras arising from quantized universal enveloping algebras, J. Amer. Math. Soc., 3 (1990), 257-296, MR 91e:17009, Zbl 0695.16006.

[Lu2] _ Introduction to Quantum Groups, Progress in Mathematics, 110, Birkhäuser Boston, Boston, MA, 1993, MR 94m:17016, Zbl 0788.17010.

[Po] V.L. Popov, Representations with a free module of covariants, Funktsional. Anal. i Prilozhen., 10 (1976), 91-92 (= Funct. Anal. Appl., 10 (1976), 242-243), MR 54 \#5255, Zbl 0365.20053.

[Ri1] R.W. Richardson, The conjugating representation of a semisimple group, Invent. Math., 54 (1979), 229-245, MR 81a:14023, Zbl 0424.20035.

[Ri2] _ An application of the Serre conjecture to semisimple algebraic groups, in 'Algebra, Carbondale 1980', R.K. Amayo, editor, Lecture Notes in Math., 848, Springer-Verlag, Berlin-Heidelberg-New York (1981), 141-151, MR 83j:20047, Zbl 0457.14022. 
[Sc] G.W. Schwarz, Representations of simple Lie groups with a free module of covariants, Invent. Math., 50 (1978), 1-12, MR 80c:14008, Zbl 0391.20033.

[SW] G.W. Schwarz and D.L. Wehlau, Invariants of four subspaces, Ann. Inst. Fourier (Grenoble), 48 (1998), 667-697, MR 99i:14055, Zbl 0899.20024.

Received December 28, 2000. The author acknowledges partial financial support from the European TMR network "Algebraic Lie representations", contract no. ERB FMRXCT97-0100.

Institut De Recherche Mathématique AvancÉe

Université Louis Pasteur et CNRS

7, Rue René Descartes

F-67084 Strasbourg Cedex

FRANCE

E-mail address: baumann@math.u-strasbg.fr 